\title{
Stellungnahme des „Expertenkreises Aerosole“: Aerosole und SARS-CoV-2 - Entstehung, Infektiosität, Ausbreitung und Minderung luftgetragener, virenhaltiger Teilchen in der Atemluft
}

\author{
Aerosols and SARS-CoV-2: Statement of the "Expert Group \\ Aerosols" on the development, infectivity, spread and reduction \\ of airborne, virus-containing particles in the air
}

Autoren

Stefan Brockmann', Achim Dittler ${ }^{2}$, Gunnar Grün³, Michael Haibel ${ }^{4}$, Thomas Iftner ${ }^{5}$, Hans-Georg Kräusslich ${ }^{6}$, Boris Mizaikoff', Jennifer Niessner ${ }^{8}$, B Richter ${ }^{9}$, Claudia Spahn ${ }^{9}$, Konstantinos Stergiaropoulos ${ }^{10}$, Heike von Baum ${ }^{11}$

Institute

1 Gesundheitsschutz und Epidemiologie, Landesgesundheitsamt Baden-Wurttemberg, Stuttgart, Deutschland

2 Institut für Mechanische Verfahrenstechnik und Mechanik - GPS, Karlsruher Institut für Technologie (KIT), Karlsruhe, Deutschland

3 Institut für Akustik und Bauphysik an der Universität Stuttgart, Universität Stuttgart, Stuttgart, Deutschland

4 Labor für Raumlufttechnik, Hochschule für angewandte Wissenschaften Biberach, Biberach, Deutschland

5 Institut für Medizinische Virologie und Epidemiologie der Viruskrankheiten, Universitätsklinikum Tübingen, Tubingen, Deutschland

6 Zentrum für Infektiologie, Abteilung Virologie , UniversitätsKlinikum Heidelberg, Heidelberg, Deutschland

7 Institut für Analytische und Bioanalytische Chemie, Universität Ulm, Ulm, Deutschland

8 Institut für Strömung in additiv gefertigten porösen Strukturen (ISAPS), Hochschule Heilbronn, Heilbronn, Deutschland

9 Freiburger Institut für Musikermedizin, Hochschule für Musik und Universitätsklinikum Freiburg, Freiburg, Deutschland

10 Institut für Gebäudeenergetik, Thermotechnik und Energiespeicherung (IGTE), Universität Stuttgart, Stuttgart, Deutschland

11 Sektion Klinikhygiene, Inst. für Med. Mikrobiologie und Hygiene, Universitätsklimikum Ulm, Ulm, Deutschland

Schlüsselwörter

SARS-CoV-2, Aerosol, Coronavirus, airborne

Key words

SARS-CoV-2, airborne, aerosol, coronavirus
Bibliografie

Gesundheitswesen 2021; 83: 231-234

DOI 10.1055/a-1363-0972

ISSN $0941-3790$

(C) 2021. Thieme. All rights reserved.

Georg Thieme Verlag KG, Rüdigerstraße 14,

70469 Stuttgart, Germany

\section{Korrespondenzadresse}

Stefan Brockmann

Gesundheitsschutz und Epidemiologie

Landesgesundheitsamt Baden-Wurttemberg

Nordbahnhofstrasse 135

70191 Stuttgart

Deutschland

stefan.brockmann@rps.bwl.de

\section{ZUSAMMENFASSUNG}

Aerosole werden gegenwärtig als einer der wesentlichen Übertragungswege für SARS-CoV-2 angesehen, doch ein umfassendes Verständnis der Prozesse und entsprechendes Handeln/ Anpassen von Schutzkonzepten erfordert den Austausch von Informationen über interdisziplinäre Grenzen hinweg. Vor diesem Hintergrund hat die baden-württembergische Landesregierung Anfang Oktober 2020 einen multidisziplinären „Expertenkreis Aerosole“ aus Ingenieuren, Naturwissenschaftlern und Medizinern ins Leben gerufen. In seiner Stellungnahme hat der Kreis den aktuellen Kenntnisstand in allen relevanten Fachrichtungen im Kontext der luftübertragenen SARS-CoV-2 Infektion zusammengetragen. Darin wird neben den allseits bekannten Hygiene- und Abstandsregeln die Bedeutung des richtigen Tragens wirksamer Masken hervorgehoben und der Eigen- sowie Fremdschutz verschiedener Masken gegenübergestellt. Weiterhin wird auf die Notwendigkeit des dynamischen und richtigen Lüftens hingewiesen und es werden exemplarisch Lüftungsintervalle und -zeiträume für unterschiedliche Szenarien illustriert. Auf die Wirksamkeit stationärer oder mobiler Innenraumluftfilter als ein wichtiger Baustein im Schutzkon- 
zept wird eingegangen. Die erste Stellungnahme des Expertenkreises macht deutlich, dass die bestehenden Hygiene- und Abstandsregeln $(\mathrm{AHA}+\mathrm{L})$ nur in ihrer Kombination und richtig angewandt, den bestmöglichen Schutz vor einer SARS-CoV-2 Infektion bieten.

\section{ABSTRACT}

Aerosols are currently seen as one of the main transmission routes for SARS-CoV-2, but a comprehensive understanding of the processes and appropriate action/adaptation of protection concepts requires the exchange of information across interdisciplinary boundaries. Against this background, the BadenWürttemberg state government launched in October 2020 a multidisciplinary “Expert Group Aerosols” comprising engi- neers, natural scientists and medical professionals. In its statement, the group has compiled the current state of knowledge in all relevant disciplines in the context of airborne SARS-CoV-2 infection. In addition to the well-known hygiene and social distancing rules, the importance of the correct use of effective masks is emphasized. Furthermore, the necessity for dynamic and correct ventilation is pointed out and illustrated with ventilation intervals and periods for different scenarios as examples. The effectiveness of stationary or mobile cabin air filters as an important component in the protection concept is discussed. The first opinion of the expert group makes it clear that the existing hygiene and social distancing rules offer the best possible protection against SARS-CoV-2 infection only when correctly applied in combination.

\section{Einleitung}

Aerosole sind kleinste $(<5 \mu \mathrm{m})$ Schwebeteilchen mit einem Gemisch aus Gas und flüssigen oder festen Teilchen, die im Gegensatz zu größeren Tröpfchen lange in der Luft verbleiben. Aerosole werden vom Menschen schon beim Atmen in hohen Konzentrationen emittiert und können bei infizierten Personen Viren enthalten und Infektionen übertragen. Aerosole werden gegenwärtig als einer der wesentlichen Übertragungswege für SARS-CoV2 angesehen, wobei derzeit nicht abschließend geklärt ist, welcher Anteil der Ansteckungen tatsächlich auf Aerosole zurückzuführen ist.

Aerosole werden in vielen Bereichen seit langem erforscht, doch die Verknüpfung zur SARS-CoV-2 Ausbreitung ist Neuland und überschneidet mehrere Fachgebiete. Entsprechend liegen vorläufige und oft widersprüchliche Erkenntnisse aus verschiedenen Perspektiven und zu verschiedensten Facetten dieses Infektionsweges vor.

Vor diesem Hintergrund hat die baden-württembergische Landesregierung Anfang Oktober 2020 einen multidisziplinären „Expertenkreis Aerosole“ aus Ingenieuren, Naturwissenschaftlern und Medizinern ins Leben gerufen. Der Expertenkreis hat den aktuellen Kenntnisstand in allen relevanten Fachrichtungen im Kontext der luftübertragenen SARS-CoV-2 Infektion zusammengetragen. Das Spektrum der Fragestellungen reicht von medizinisch/virologischen Aspekten bezüglich des Vorhandenseins von Viren und des Erhalts der Infektiosität in Aerosolen bis hin zu ingenieurrelevanten/naturwissenschaftlichen Erkenntnissen zur Ausbreitung und Minderung luftgetragener, feinster (potentiell infektiöser) Teilchen in der Atemluft. Auf dieser Grundlage wurde eine übergreifende Bewertung von Schutzkonzepten vorgenommen, mit dem Ziel, Quellen virusbeladener Luft zu vermeiden oder zu verringern und Senken zu stärken.

Eine erste gemeinsame Stellungnahme zum luftgetragenen SARSCoV-2-Infektionsweg wurde im Dezember 2020 fertiggestellt. Diese Langversion der Stellungnahme ist in 3 Teile gegliedert:

I. Virologische und medizinische Aspekte des Vorhandenseins von Viren und des Erhalts ihrer Infektiosität in Aerosolen

II. Ingenieurwissenschaftliche/naturwissenschaftliche Aspekte der Ausbreitung und Minderung gasgetragener Partikeln

III. Übergreifende Fragestellungen
Nachfolgend findet sich eine verkürzte Version der originalen Stellungnahme. Die Stellungnahme in der Langversion ist im Internet abrufbar [1].

\section{Aerosol als Übertragungsweg für SARS-CoV-2}

Aerosole, also gasgetragene flüssige oder feste Partikeln, werden gegenwärtig als einer der wesentlichen Übertragungswege für SARS-CoV2 angesehen, wobei derzeit nicht abschließend geklärt ist, welcher Anteil der Ansteckungen tatsächlich auf Aerosole zurückzuführen ist. Durch Atmen, Sprechen, Singen oder Schreien sowie durch Husten und Niesen emittieren Lebewesen Tröpfchen bzw. exhalieren zuvor eingeatmeten Feinstaub. Die mit der Luft ausgeatmeten Partikeln können dabei das Virus tragen. Die exhalierten Partikeln haben - je nach Aktivität - eine unterschiedliche Größe und werden mit unterschiedlicher Rate/in unterschiedlicher Konzentration ausgestoßen. Tropfen verdunsten - abhängig von ihrer Zusammensetzung, Größe und dem Zustand der umgebenden Luft (Sättigung) - unterschiedlich schnell. Zurück bleiben feinste Nuklei, die sich sehr schnell um die Quelle und in (geschlossenen) Räumen verteilen.

Eine Infektion kann immer auftreten, wenn infektiöses Virus auf die Schleimhäute des Atemtraktes einer empfänglichen Person trifft; je größer die Menge an infektiösen Viren, die eintritt, desto wahrscheinlicher wird die Infektion, zumindest im Mittel. Insofern sind sowohl die Konzentration der infektiösen Viren als auch die Expositionsdauer von entscheidender Bedeutung, eine kritische Konzentration oder Zeit, bei deren Unterschreiten kein Risiko bestünde, lässt sich jedoch nicht angeben. Ebenso wenig kann man eine direkte Korrelation von Virusmenge und Dauer der Exposition angeben. Es gibt aber Hinweise darauf, dass bei Exposition einer großen Virusmenge sowie in Abhängigkeit von der Penetrationstiefe im Atemwegstrakt (Partikeln $<5 \mu$ m penetrieren tief in die Atemwege) der Krankheitsverlauf schwerer sein kann.

\section{Schutzmaßnahmen im Außenbereich}

Die Ansteckungsgefahr durch Aerosole kann durch das Atmen sauberer, nicht mit Viren belasteter, Atemluft vermindert werden. Saubere - also nicht infektiöse - Atemluft ist in der Regel in gut durch- 
mischter Außenluft bei genügend großem Abstand zu potentiell infektiösen Quellen vorhanden. Kommen sich Menschen näher, erhöht sich die Wahrscheinlichkeit, dass man Exhalat anderer einatmet. Daher empfiehlt sich im Außenbereich:

- Einhaltung großer Abstände zu anderen Menschen - z. B. durch Wegführungen/Zonierungen

- Aufenthaltsdauer in der Nähe von Menschen reduzieren - z. B. durch Zugangs- und Aufenthaltskonzepte

- Wo Abstände nicht eingehalten werden können, empfiehlt sich das korrekte Tragen einer möglichst wirksamen Maske

\section{Schutzmaßnahmen im Innenbereich}

In der kälteren Jahreszeit finden viele Zusammenkünfte von Menschen in Innenräumen statt. Hier kommt die Qualität der Atemluft in Innenräumen zum Tragen. Wesentlicher Baustein, ein Infektionsrisiko über Aerosole zu mindern, ist dabei die Sicherstellung sauberer Atemluft im Innenraum. Die Zufuhr von Außenluft über RLT-Anlagen oder Fensterlüftung/die Abscheidung von Partikeln stellen den wirksamsten Weg der Minderung der Konzentration potentiell infektiöser Partikeln in der Atemluft dar. Dies bedeutet:

- Ein möglichst häufiger Luftaustausch (Außenluftzufuhr) ist sicherzustellen - Richtwerte für verschiedene Nutzungsszenarien (wie z. B. Büro, Klassenzimmer oder Hörsaal) finden sich in Tabelle 6-10 der Langfassung [1]. In > Tab. 1 ist der Volumenstrom bei Fensterlüftung in Abhängigkeit der Außentemperatur dargestellt.

- Dieser kann durch richtiges, regelmäßiges Fenster-Lüften erfolgen

- Ab einer Konzentration von 800 ppm $\mathrm{CO}_{2}$ sollte gelüftet werden

- Zur Ermittlung des Zeitpunkts eines Lüftens und zur Überprüfung des Lüftungs-Erfolgs eignen sich genaue $\mathrm{CO}_{2}$-Messgeräte

- Stoß- und Querlüften stellen die effizientesten Arten der Fenster-Lüftung dar

- Alle Fenster weit öffnen („Kippen“ ist nicht „Lüften“)

- Die Lüftungsdauer richtet sich nach der Raumgröße, der Anzahl der Fenster und dem Temperaturunterschied zwischen „innen“ und „außen“. Exemplarische Lüftungsdauern bei einer Raumgröße von $40 \mathrm{~m}^{2}$ finden sich in > Tab. 2 . Berechnungen für weitere Raumgrößen finden sich in der Langfassung.
Je niedriger die Außentemperatur desto höher ist der Volumenstrom. Je mehr Fenster geöffnet werden, desto höher ist der Volumenstrom.

In Zeiten, in denen Räume nicht ausreichend gelüftet werden können oder in Situationen, die bspw. durch viele Personenwechsel gekennzeichnet sind, können hoch wirksame, mobile Innenraumluftfilter einen zusätzlichen Baustein zur Minderung der Partikel-konzentration in geschlossenen Innenräumen darstellen.

Zusätzlich können auch Technologien der Reinigung der Luft unter Voraussetzung eines Wirksamkeitsnachweises Mittel sein, um die Konzentration potentiell infektiöser Partikeln im Aerosol zu senken.

Lüften - oder auch Filtern/Reinigen der Luft - mindert jedoch alleine nicht das Risiko einer Infektion durch kurzräumige Exposition (bspw. durch direktes Anhusten). Daher sollten neben Lüften und Filtern/Reinigen auch in Innenräumen:

- Die Anzahl möglicher Quellen (Anzahl an Personen) reduziert werden

- Abstände zu möglichen Quellen maximiert werden

- Die Aufenthaltsdauern möglichst kurzgehalten werden

- Ausreichend groß dimensionierte Plexiglas-Trennwände zur Minderung kurzzeitig kleinräumiger Exposition nach Möglichkeit in bestimmten Situationen vorgesehen werden (z. B. in KassenBereichen mit ständig wechselndem Kundenkontakt)

- Wirkungsvolle Masken zur Sicherstellung eines bestmöglichen Fremd- wie Eigenschutzes richtig getragen werden.

Das korrekte Tragen einer wirkungsvollen Maske ist im AHA + LKonzept ein essentieller Schutzbaustein, dessen Wirkung gesteigert werden kann, wenn statt der nicht medizinischen Alltagsmasken bspw. sog. MNS (nach DIN/EN 14683), FFP2-, KN95- oder N95Halbmasken getragen werden.

\section{Virenquellen}

Bei den Virenquellen unterscheiden wir zwischen Primärquellen (Personen) und Sekundärquellen (Oberflächen, auf denen sich Tröpfchen abgeschiedenen haben), die aus diversen Gründen wieder aufgewirbelt werden können. Im Folgenden fokussieren wir uns

> Tab. 1 Volumenstrom in einen Raum über Drehfenster (1,8 $\mathrm{m}^{2}, 90^{\circ}$ Öffnung) bei unterschiedlichen Außentemperaturen.

\begin{tabular}{|c|c|c|c|c|c|c|}
\hline \multicolumn{7}{|c|}{$V_{\text {ist }}$ in $m^{3} / h$} \\
\hline & & $15^{\circ} \mathrm{C}$ & $10^{\circ} \mathrm{C}$ & $0^{\circ} \mathrm{C}$ & $-5^{\circ} \mathrm{C}$ & $-10^{\circ} \mathrm{C}$ \\
\hline \multirow{5}{*}{ Fenster-anzahl } & 1 & 646 & 842 & 1000 & 1258 & 1369 \\
\hline & 2 & 1292 & 1684 & 2000 & 2516 & 2738 \\
\hline & 3 & 1938 & 2525 & 3000 & 3774 & 4107 \\
\hline & 4 & 2584 & 3367 & 4000 & 5032 & 5476 \\
\hline & 5 & 3230 & 4209 & 5000 & 6290 & 6844 \\
\hline
\end{tabular}

> Tab. 2 Zeitspanne in Minuten (offene Fenster) für einen $40 \mathrm{~m}^{2}$ großen Raum, um das Raumluftvolumen einmal auszutauschen.

\begin{tabular}{|l|l|l|l|l|l|l|l|}
\hline & & $\mathbf{1 5}{ }^{\circ} \mathbf{C}$ & $\mathbf{1 0}^{\circ} \mathbf{C}$ & $\mathbf{5}^{\circ} \mathbf{C}$ & $\mathbf{0}^{\circ} \mathbf{C}$ & $-\mathbf{5}^{\circ} \mathbf{C}$ & $-\mathbf{1 0}^{\circ} \mathbf{C}$ \\
\hline \multirow{5}{*}{ Fenster-anzahl } & 1 & 11 & 9 & 7 & 6 & 6 & 5 \\
\cline { 2 - 9 } & 2 & 6 & 4 & 4 & 3 & 3 & 3 \\
\cline { 2 - 9 } & 3 & 4 & 3 & 2 & 2 & 2 & 1 \\
\cline { 2 - 9 } & 4 & 3 & 2 & 2 & 2 & 1 \\
\hline
\end{tabular}


\Tab. 3 Fremd- und Eigenschutz gebräuchlicher Halbmasken, von links nach rechts geordnet nach zunehmendem Eigenschutz.

\begin{tabular}{|c|c|c|c|c|c|c|c|c|c|}
\hline & & $\begin{array}{l}\text { Alltags- } \\
\text { maske }\end{array}$ & MNS & FFP1 & $\begin{array}{l}\text { FFP2/3 mit } \\
\text { Ventil }\end{array}$ & FFP2 & KN95 & N95 & FFP3 \\
\hline & Prüfung & keine & EN 14683 & EN 149 & EN 149 & EN 149 & GB 2626 & $\begin{array}{l}42 \text { CFR } \\
\text { Part } 84\end{array}$ & EN 149 \\
\hline \multirow[t]{3}{*}{ Fremdschutz } & Minderung Auswurfweite & ja & ja & ja & ja & ja & ja & ja & ja \\
\hline & Wirkung als Spuckschutz & ja & ja & ja & ja & ja & ja & ja & ja \\
\hline & $\begin{array}{l}\text { Minderung Ausatmen feiner } \\
\text { Partikel }(<1 \mu \mathrm{m})\end{array}$ & $10-70 \%$ & $40-90 \%$ & $>80 \%$ & nein & $>94 \%$ & $>95 \%$ & $>95 \%$ & $>99 \%$ \\
\hline Eigen- & $\begin{array}{l}\text { Minderung Einatmen feiner } \\
\text { Partikel (<1 } \mu \mathrm{m})\end{array}$ & $10-70 \%$ & $40-90 \%$ & $>80 \%$ & $>94 / 99 \%$ & $>94 \%$ & $>95 \%$ & $>95 \%$ & $>99 \%$ \\
\hline
\end{tabular}

auf Primärquellen. In Bezug auf die Ermittlung der Bedeutung von Sekundärquellen besteht weiterer Forschungsbedarf.

Primärquellen können gemindert werden durch die Limitierung der Anzahl Personen und der Aufenthaltsdauer in einem Raum (Zugangskontrolle), durch Abstand (Vermeidung von direkter Exposition) und durch das Tragen von Masken ( $\triangleright$ Abb. 1 Langfassung)

\section{Halbmasken}

Masken reduzieren die ein- und ausgeatmete Konzentration an Partikeln und dienen dadurch dem Fremd- und Eigenschutz. Generell ist darauf zu achten, eine möglichst wirkungsvolle Maske zu verwenden und diese richtig zu tragen, d.h. sie muss Mund und Nase vollständig bedecken und eng anliegen. Leckagen und Bypass-Strömungen können die Schutzwirkung drastisch mindern ( $>$ Abb. 2 Langfassung)

Masken lassen sich - abhängig vom Abscheidegrad gegenüber Partikeln - in verschiedene Kategorien einteilen. Man unterscheidet Fremd- und Eigenschutz und differenziert bezüglich des Abscheidungsvermögens größerer $(>1 \mu \mathrm{m})$ und feinster $(<1 \mu \mathrm{m})$ Partikeln. > Tab. 3 zeigt eine Übersicht.

Visiere und Plexiglaswände dienen der Abscheidung großer Tröpfchen und beeinflussen die Ausbreitungsrichtung sowie die lokale Konzentration von Partikeln $<1 \mu \mathrm{m}$ im Raum. Damit dienen sie bei kurzzeitigem Kontakt für größere Tröpfchen als „Spuck- und Spritzschutz" und für Partikeln $<1 \mu$ m als Strömungshindernis. Die Luft mit den darin enthaltenen kleinen Aerosolpartikeln kann allerdings mit der Zeit um das Strömungshindernis herum strömen, sodass die globale Aerosolkonzentration im Raum durch Visiere und Plexiglaswände über sehr lange Zeit nicht beeinflusst wird. Beide Vorrichtungen ändern also kurzfristig und kurzräumig die Aerosolverteilung, nicht aber die langfristige Verteilung und ebenso wenig die Gesamtanzahl an kleinen Aerosolpartikeln im Raum.

Einwegmasken sind ordnungsgemäß über den Restmüll zu entsorgen. Bei Mehrwegmasken ist auf fachgerechte Sterilisation zu achten, da bei nicht sachgerechter Durchführung Gefahr von Sekundäremission besteht.

Zusätzlich sollten die (bekannten) Hygiene- und Verhaltensregeln beachtet werden:

- Hände ausreichend lange waschen und ggf. desinfizieren

- Nies- und Husten-Etikette einhalten (z. B. in die Armbeuge)

- Flächen geeignet reinigen (Insbesondere Flächen, die häufig berührt werden, regelmäßig reinigen. Für Risikobereiche wie Krankenhäuser, Pflegeheime etc. liegen diesbezügliche Desinfektionspläne bereits vor.)

- Bei Erkrankungszeichen
- auf den Besuch von chronisch kranken, abwehrgeschwächten Personen verzichten

- Kinder nicht in die Schule, Kita schicken

- Erwachsene: nicht zur Arbeit gehen

Vulnerable Personengruppen sind besonders schutzbedürftig. Hier kommen im medizinischen und öffentlichen Bereich sowohl organisatorisch-logistische als auch baulich-technische Schutzmaßnahmen zum Tragen. In den Tabellen 13-16 der Langfassung [1] sind diese Schutzmaßnahmen ausführlich und nach folgenden Personengruppen bzw. der räumlichen Nutzung differenziert dargestellt:

- Organisatorisch-logistische Maßnahmen im Medizinischen Bereich (Tabelle 13)

- Baulich-technische Maßnahmen im Medizinischen Bereich (Tabelle 14)

- Organisatorisch-logistische Maßnahmen im Öffentlichen Raum (Tabelle 15)

- Baulich-technische Maßnahmen im Öffentlichen Raum (Tabelle 16)

Zusammenfassend lässt sich festhalten: Die konsequente Einhaltung der Kombination einer Vielzahl von wirksamen Einzelmaßnahmen mindert das Risiko einer möglichen Infektion durch belastete Aerosole bestmöglich.

\section{Danksagung}

Wir danken Sabine Gerbersdorf und Caroline Liepert für die Unterstützung des Expertenkreises und Sabine Gerbersdorf für die kritische Durchsicht und Anregungen bei der Erstellung des Manuskripts.

Interessenkonflikt

Die Autorinnen/Autoren geben an, dass kein Interessenkonflikt besteht.

Literatur

[1] Stellungnahme des Expertenkreis Aerosole: Aerosole und SARS-CoV-2 - Entstehung, Infektiosität, Ausbreitung \& Minderung luftgetragener, virenhaltiger Teilchen in der Atemluft: (04.12.2020). Im Internet: https://mwk.baden-wuerttemberg.de/fileadmin/redaktion/m-mwk/ intern/dateien/Anlagen_PM/20201204_Stellungnahme_Aerosole_ SARSCoV-2.pdf; Stand: 16.12.2020 